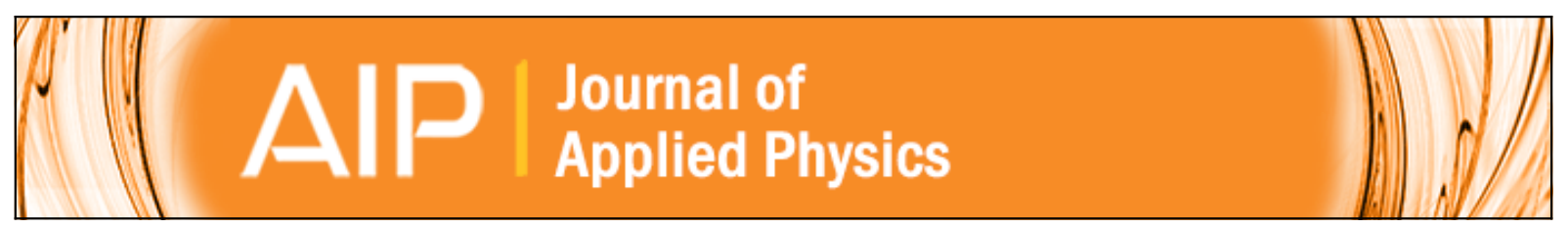

\title{
Electric properties and carrier multiplication in breakdown sites in multi-crystalline silicon solar cells
}

Matthias Schneemann, Thomas Kirchartz, Reinhard Carius, and Uwe Rau

Citation: Journal of Applied Physics 117, 205703 (2015); doi: 10.1063/1.4921286

View online: http://dx.doi.org/10.1063/1.4921286

View Table of Contents: http://scitation.aip.org/content/aip/journal/jap/117/20?ver=pdfcov

Published by the AIP Publishing

\section{Articles you may be interested in}

Response to "Comment on 'Origin of breakdown mechanism in multicrystalline silicon solar cells"' [Appl. Phys. Lett. 102, 246101 (2013)]

Appl. Phys. Lett. 102, 246102 (2013); 10.1063/1.4811550

Comment on "Origin of breakdown mechanism in multicrystalline silicon solar cells" [Appl. Phys. Lett. 101, 093903 (2012)]

Appl. Phys. Lett. 102, 246101 (2013); 10.1063/1.4811549

Origin of breakdown mechanism in multicrystalline silicon solar cells

Appl. Phys. Lett. 101, 093903 (2012); 10.1063/1.4749821

Understanding junction breakdown in multicrystalline solar cells

J. Appl. Phys. 109, 071101 (2011); 10.1063/1.3562200

Nanoscale measurements of local junction breakdown in epitaxial film silicon solar cells

Appl. Phys. Lett. 97, 092107 (2010); 10.1063/1.3479534

Frustrated by

old technology?

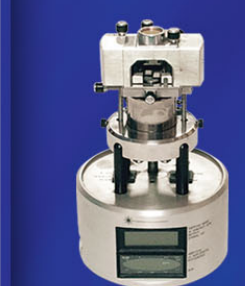

Is your AFM dead

and can't be repaired?

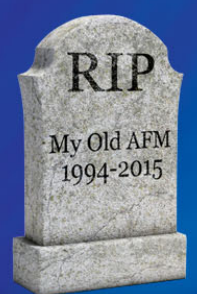

Sick of bad customer support?

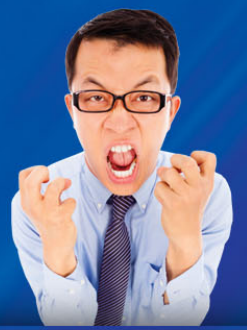

It is time to upgrade your AFM Minimum $\$ 20,000$ trade-in discount for purchases before August 31st

Asylum Research is today's technology leader in AFM

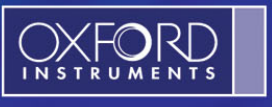




\title{
Electric properties and carrier multiplication in breakdown sites in multi-crystalline silicon solar cells
}

\author{
Matthias Schneemann, ${ }^{1}$ Thomas Kirchartz, ${ }^{1,2, a)}$ Reinhard Carius, ${ }^{1}$ and Uwe Rau ${ }^{1}$ \\ ${ }^{1}$ IEK5-Photovoltaics, Forschungszentrum Jülich, Jülich 52425, Germany \\ ${ }^{2}$ Faculty of Engineering and CENIDE, University of Duisburg-Essen, Carl-Benz-Str. 199, Duisburg 47057, \\ Germany
}

(Received 1 December 2014; accepted 6 May 2015; published online 27 May 2015)

\begin{abstract}
This paper studies the effective electrical size and carrier multiplication of breakdown sites in multi-crystalline silicon solar cells. The local series resistance limits the current of each breakdown site and is thereby linearizing the current-voltage characteristic. This fact allows the estimation of the effective electrical diameters to be as low as $100 \mathrm{~nm}$. Using a laser beam induced current (LBIC) measurement with a high spatial resolution, we find carrier multiplication factors on the order of 30 (Zener-type breakdown) and 100 (avalanche breakdown) as new lower limits. Hence, we prove that also the so-called Zener-type breakdown is followed by avalanche multiplication. We explain that previous measurements of the carrier multiplication using thermography yield results higher than unity, only if the spatial defect density is high enough, and the illumination intensity is lower than what was used for the LBIC method. The individual series resistances of the breakdown sites limit the current through these breakdown sites. Therefore, the measured multiplication factors depend on the applied voltage as well as on the injected photocurrent. Both dependencies are successfully simulated using a series-resistance-limited diode model. (C) 2015 AIP Publishing LLC. [http://dx.doi.org/10.1063/1.4921286]
\end{abstract}

\section{INTRODUCTION}

Breakdown in solar cells and-modules may be harmful for affected devices, possibly leading to the destruction of the cell or module. A likely scenario is the partial shading of a module. In this case, the fully illuminated solar cells in a string build up a photovoltage that will then be applied as a negative bias to a shaded cell connected in series. Therefore, intense research into the reasons and the physical understanding of breakdown were undertaken, especially for (multi-) crystalline silicon solar cells. ${ }^{1-8}$ Like in other silicon-based electronic devices, breakdown causes the emission of visible light, the reverse biased electroluminescence (ReBEL). ${ }^{9,10}$ It is therefore possible to investigate and locate the occurring high currents not only with (Lock-in) thermography $^{2}$ (LIT) but also with CCD cameras offering higher spatial resolution.

For multi-crystalline silicon solar cells, three classes of defects causing breakdown were identified: aluminum contamination on the cell surface ${ }^{4}$ (type-1), iron silicide precipitates in grain boundaries ${ }^{5,6,11}$ (type-2), and strongly curved $p$ - $n$-junctions (type-3). The latter being caused by either etch pits in acidly etched cells ${ }^{7}$ or at sites of preferred phosphorous diffusion in grain boundaries of cells with alkaline texture. ${ }^{12} \mathrm{~A} p$ - $n$-junction with a radius of curvature of around $300 \mathrm{~nm}$ was shown to reduce the breakdown voltage to around $V_{\mathrm{O}}=-12 \quad \mathrm{~V}^{13} \quad$ at a doping concentration $N_{\mathrm{D}}=10^{16} \mathrm{~cm}^{-3}$ ). For the breakdown types 1 and 2 , the excitation of the carriers was attributed to Zener tunneling or thermionic field emission. Using carefully calibrated

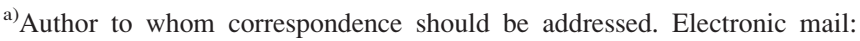
t.kirchartz@fz-juelich.de
}

emission spectra, the light generation process was recently shown to originate from phonon-assisted intra-band transitions of holes and electrons within the valence- and conduction bands, respectively. ${ }^{14}$

The determination of the breakdown mechanism, i.e., avalanche breakdown or Zener tunneling was approached via light intensity-voltage characteristics ${ }^{8}(\Phi-V$ characteristics) and new thermography methods (temperature coefficient dark LIT, TC-DLIT, and multiplication factor illuminated LIT, MF-ILIT). ${ }^{2}$ While $\Phi-V$ characteristics of macroscopic regions measured by a CCD-camera indeed showed "hard" and "soft" exponential behavior, which were interpreted as avalanche- and Zener-type breakdown, respectively, microscopic investigations falsified those conclusions. ${ }^{15} \Phi-V$ characteristics of all individual breakdown sites are linearized by a local series resistance, such that the exponential nature of the reverse current is only visible in the very early stages of the breakdown. Thus, a growing number of spots in a larger field of view will yield a superlinear regional $\Phi-V$ characteristic. The origin of the series resistance is expected to be current crowding ${ }^{8,16,17}$ towards (and from) the defect. While earlier investigations ${ }^{4,14}$ unveiled that the spot sizes are smaller than $1 \mu \mathrm{m}$, i.e., below the resolution of an optical set-up, the present work analyses a large number of spots of all types by a simple model and finds typical radii on the order of $100 \mathrm{~nm}$.

Another approach to identify the breakdown mechanism is measuring the local current amplification with the MFILIT technique. ${ }^{2}$ In Ref. 2, the injected light generated current was multiplied up to a factor of 4 in certain cell areas. This observation was considered to be proof of avalanche breakdown. However, the used spatial resolution of 
$640 \times 512$ pixels applied to a $(15.6 \mathrm{~cm})^{2}$ solar cell area is equivalent to an area of $(300 \mu \mathrm{m})^{2}$ per pixel. Taking the above mentioned defect size into account, most of the pixel area is not covered by breakdown sites and therefore does not contribute to the amplification of the injected current, implying that the values determined using MF-ILIT must necessarily be much too low. This is of particular relevance, if these results are used as evidence against avalanche multiplication in type- 2 breakdown sites. The present work overcomes the limitation of spatial resolution by applying a microscopically focused laser beam, which is scanning the area of a breakdown site while recording the generated current (laser beam induced current (LBIC)).

The present publication shows that three points have to be considered in order to measure a current amplification(i) The reverse voltage must be sufficiently high to allow breakdown to happen locally; (ii) the current through the defect must not have reached its series resistance limitation; and finally (iii) the photocurrent must be as low as possible to avoid disturbing the local physics, i.e., if the light beam induced current is high enough to push the defect into series resistance limitation, the measured current multiplication will be reduced. The highest multiplication factors, we were able to measure, are around 30 for type- 2 and 100 for type- 3 spots. Though the initial breakdown mechanism of type-2 spots may indeed be Zener tunneling, but it is certainly followed by carrier multiplication.

\section{EFFECTIVE ELECTRICAL DEFECT SIZE}

To obtain the local currents of the individual breakdown sites, we measured the global ReBEL intensity $\Phi_{\mathrm{G}}$ of a sample with a camera at different applied voltages $V$ while recording also the respective global current $I$. After an onset phase, the global current $I$ is linearly correlated to the global ReBEL intensity $\Phi_{G}$ of the cell [Fig. 1(a)]. This correlation allows us to view the ReBEL intensity as a measure for the local electrical current and individual breakdown currents $I_{\mathrm{S}}$ are obtained using the ratio $I / \Phi_{\mathrm{G}}$. The series resistance limited $\Phi-V$ characteristics of individual spots yield local currents up to $I=0.6 \mathrm{~mA}$, which correspond to 100 intensity units in Fig. 1(b). The inverse slope $\Delta V / \Delta I$ of the depicted $I_{\mathrm{S}}-V$ characteristics equates to the individual series resistance of the breakdown site $R_{\mathrm{S}}$.

We therefore measured typical breakdown site series resistances $R_{\mathrm{S}}$ ranging between $10 \mathrm{k} \Omega \leq R_{\mathrm{S}} \leq 40 \mathrm{k} \Omega$. The physics of the present breakdown site is similar to the current crowding or spreading resistance at small electric contacts. A hemisphere with a radius $r$ (defect) embedded in semiinfinite solid ( $p$-type base with resistivity $\rho_{\mathrm{B}}=1 \Omega \mathrm{cm}$ ) has a contact resistance ${ }^{8,16}$

$$
R_{\mathrm{S}}=\frac{\rho_{\mathrm{B}}}{2 \pi r} .
$$

The other hemi-sphere in the emitter can be neglected because of the much lower resistivity of the material with the higher doping density $\left(\rho_{\mathrm{E}}=0.006 \Omega \mathrm{cm}\right) .{ }^{18}$ The estimated defect radii consequently range between $40 \mathrm{~nm} \leq r_{\mathrm{d}} \leq 160 \mathrm{~nm}$.
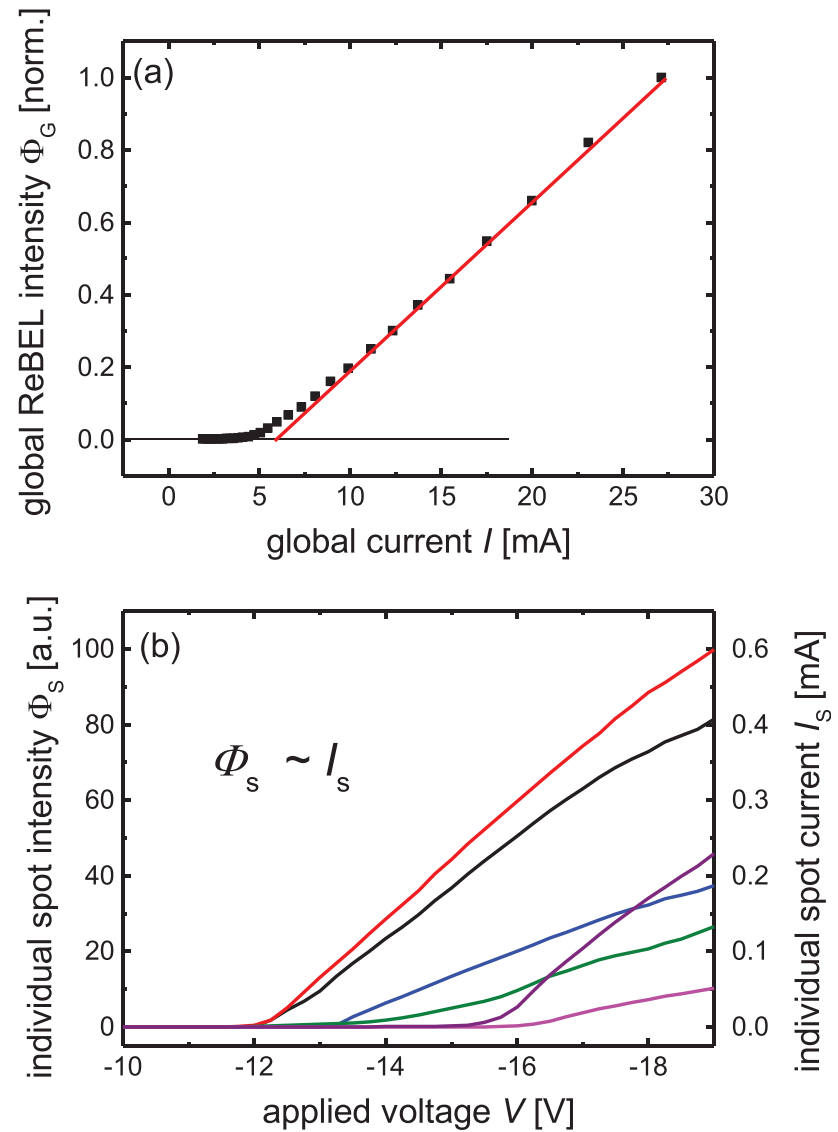

FIG. 1. (a) After a start phase the global ReBEL intensity $\Phi$ and the global cell current $I$ correlate linearly, which is also assumed to hold on the microscopic scale. It is therefore possible to calculate the local breakdown currents $I_{\mathrm{S}} \sim \Phi_{\mathrm{S}}$ using the ReBEL spot intensities $\Phi_{\mathrm{S}}(\mathrm{b})$. For the depicted spots, 100 intensity units correspond to $I_{\mathrm{S}}=0.6 \mathrm{~mA}$. (b) Reprinted with permission from Schneemann et al., Phys. Status Solidi A 207, 2597 (2010). Copyright 2010 John Wiley and Sons. ${ }^{15}$

\section{ANALYSIS OF CARRIER MULTIPLICATION}

\section{A. Experimental set-up}

We measure the carrier multiplication using an LBIC setup based on a HeNe-Laser with a wavelength of $\lambda=633 \mathrm{~nm}$. The light source is focused with a $50 \times$ microscope lens, and the samples are mounted on a computer controlled scanning table. With an applied voltage $V \leq-10 \mathrm{~V}$, we usually observe a reverse current around $100 \mathrm{~mA}$ (sample size $\leq 10 \mathrm{~cm}^{2}$ ), which is also quite noisy. Therefore, the comparably low additional photocurrent $I_{\mathrm{Ph}}$ was measured with a lock-in amplifier.

Fig. 2(a) depicts the measuring process with the modulated laser focused on the sample with a FWHM radius of $1 \mu \mathrm{m}$. As discussed in Sec. II, the radius of the defect is between $40 \mathrm{~nm} \leq r_{\mathrm{d}} \leq 160 \mathrm{~nm}$. For simplicity reasons, we assume that carriers generated directly above and below the defect are diffusing towards the breakdown site and are also accelerated in its high electric field. This cylinder is called "electrically active region" in Fig. 2(a). Consequently, only the share $a$ of the total photocurrent $I_{\mathrm{Ph}}$ is amplified. Figure 2(b) shows a suitable equivalent circuit describing the situation. The breakdown site is represented by a diode in the 

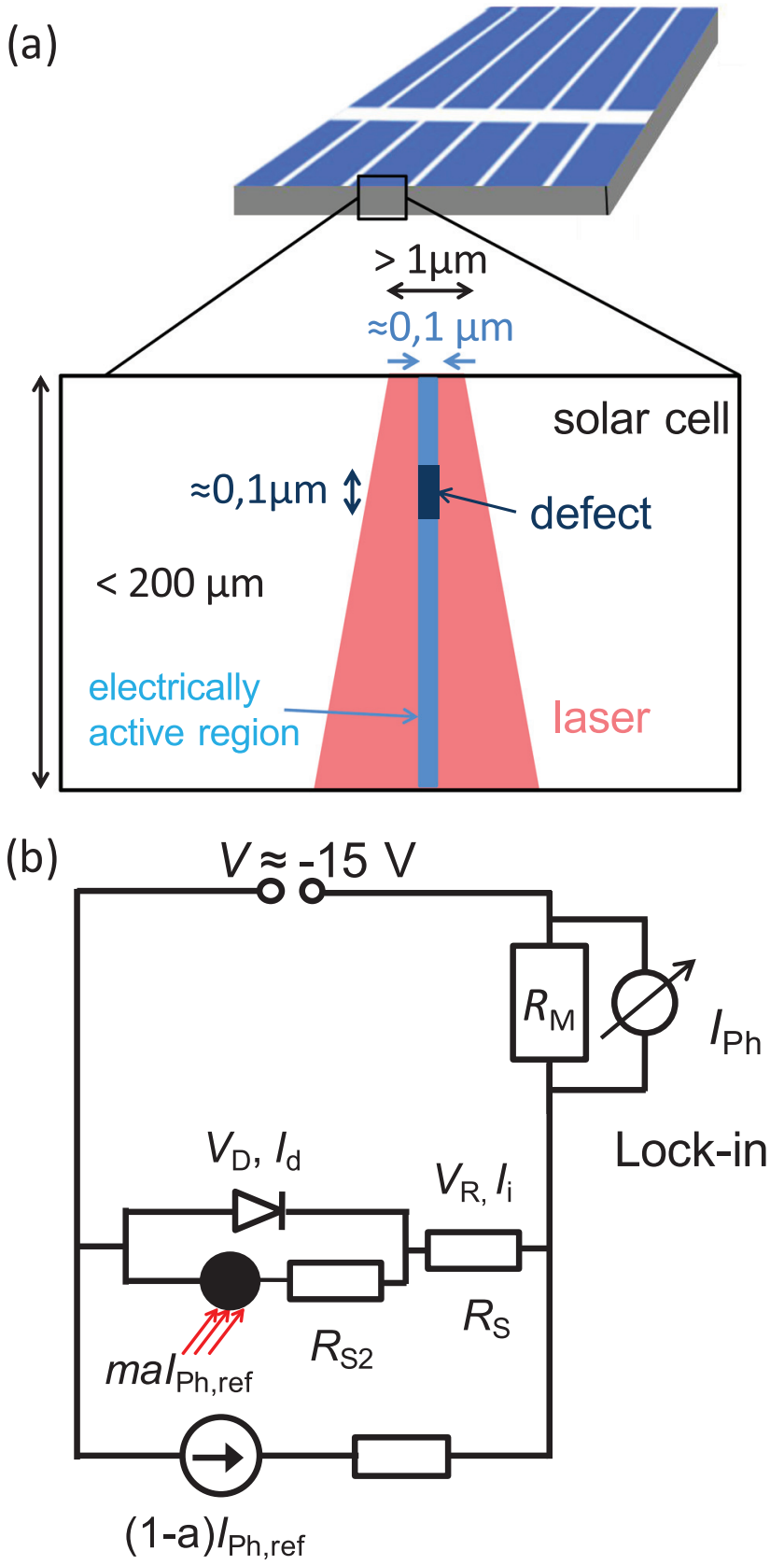

FIG. 2. (a) Origin of the measured data: the electrically active region is assumed to be a cylinder with $\approx 100 \mathrm{~nm}$ diameter, ranging over the entire depth of the cell. This implies that carriers generated above and below the defect are also forced to transit the high electric field region. The laser is focused on the surface with a spot size $\geq 1 \mu \mathrm{m}^{2}$. The illuminated area is therefore bigger than the active area. Consequently, most photons are not multiplied, and the measured multiplication factor is just a lower limit. (b) The equivalent circuit diagram of the experiment setup. An external power source holds the system at a constant voltage $V$. In the dark, the external voltage $V$ is divided into the defect voltage $V_{\mathrm{D}}$ and the voltage drop $V_{\mathrm{R}}$ over the series resistance $R_{\mathrm{S}}$. Under illumination, the share $a$ of the photogenerated current is multiplied in the defect (filled circle), being limited by an additional series resistance $R_{\mathrm{S} 2}$. The rest $(1-a)$ of the laser light is absorbed in the second arm, which is assumed to be defect free. The voltage drop, generated by the current flowing over the resistor $R_{\mathrm{M}}$, is measured with a lock-in amplifier.

dark and an additional branch under illumination that represents the photocurrent that is amplified with the factor $m$ in the breakdown site. The current $I_{\mathrm{d}}$ through the diode in the dark and the multiplied share of the photocurrent $m a I_{\mathrm{Ph}, \mathrm{ref}}$ add up to the current $I_{\mathrm{i}}$ through the defect under illumination $\left(I_{\mathrm{i}}=\mathrm{maI}_{\mathrm{Ph}, \mathrm{ref}}+I_{\mathrm{d}}\right)$. Here, $I_{\mathrm{Ph} \text {,ref }}$ is the reference photocurrent measured in a defect free region.

In parallel to the current through the defect, there exists a current not flowing through the defect. This current is assumed to be negligible in the dark. Therefore, only the photocurrent in this area is considered, and it is given by $(1-a) I_{\mathrm{Ph} \text {,ref }}$. The total photocurrent is therefore given by the sum over all branches minus the dark current, i.e.,

$$
I_{\mathrm{Ph}}=I_{\mathrm{i}}-I_{\mathrm{d}}+(1-a) I_{\mathrm{Ph}, \text { ref }} .
$$

It has to be noted that the currents are all assumed exponential functions of the voltage, and they are limited by series resistances that are shown in Figure 2(b). In order to study carrier multiplication, we can define an empirical, i.e., measured multiplication factor $M$ and try to determine the microscopic multiplication factor $m$ in the breakdown site from it. This empirical multiplication factor could be defined as the ratio of two easily measurable quantities as

$$
M=\frac{I_{\mathrm{ph}}}{I_{\mathrm{ph}, \mathrm{ref}}} .
$$

We can now calculate the dependence of $M$ on $m$ by inserting Eq. (2) and the definition for $I_{\mathrm{i}}$ into Eq. (3). We then obtain

$$
M=\frac{m a I_{\mathrm{Ph}, \mathrm{ref}}+(1-a) I_{\mathrm{Ph}, \mathrm{ref}}}{I_{\mathrm{Ph}, \mathrm{ref}}}=1+a(m-1) .
$$

The exact location of the breakdown site is found with an $x-y-s c a n$ of the approximate area. We restrained ourselves to a step size of $0.5 \mu \mathrm{m}$, which is approximately half the laser spot size.

\section{B. Multiplication factor vs. applied voltage}

Any additional photogenerated current increases the voltage drop $V_{\mathrm{R}}$ over the series resistance $R_{\mathrm{S}}$, thus reducing (for constant applied voltage $V$ ) the defect voltage $V_{\mathrm{D}}$, which determines the multiplication factor $M$. Consequently, (a) the laser intensity should be as low as possible and (b) the applied voltage $V$ should be low enough, preventing the breakdown current from being completely series resistance limited.

Figure 3(c) shows the ReBEL image (applied voltage $V=-15 \mathrm{~V}$ ) of a type-2 spot series on an alkaline etched mcSi cell compared to the correspondent representations of the LBIC measurements taken at applied voltages of $V=-11.5 \mathrm{~V}$ (b) and $V=-15 \mathrm{~V}$ (a). The light generated current in Figs. 3(a) and 3(b) was divided by a reference photocurrent $I_{\mathrm{Ph}, \text { ref }}$ taken from an inactive area on the same crystal grain, yielding the measured multiplication factor $M$. When the ReBEL image (c) is compared to the LBIC result taken at the same applied voltage (a), only a weak correlation is observed, with the strongest LBIC signal at locations of weak ReBEL intensity. A look at the intensity-voltage characteristics of the brighter spot would show that their current is already series resistance limited. We therefore reduced the applied voltage 


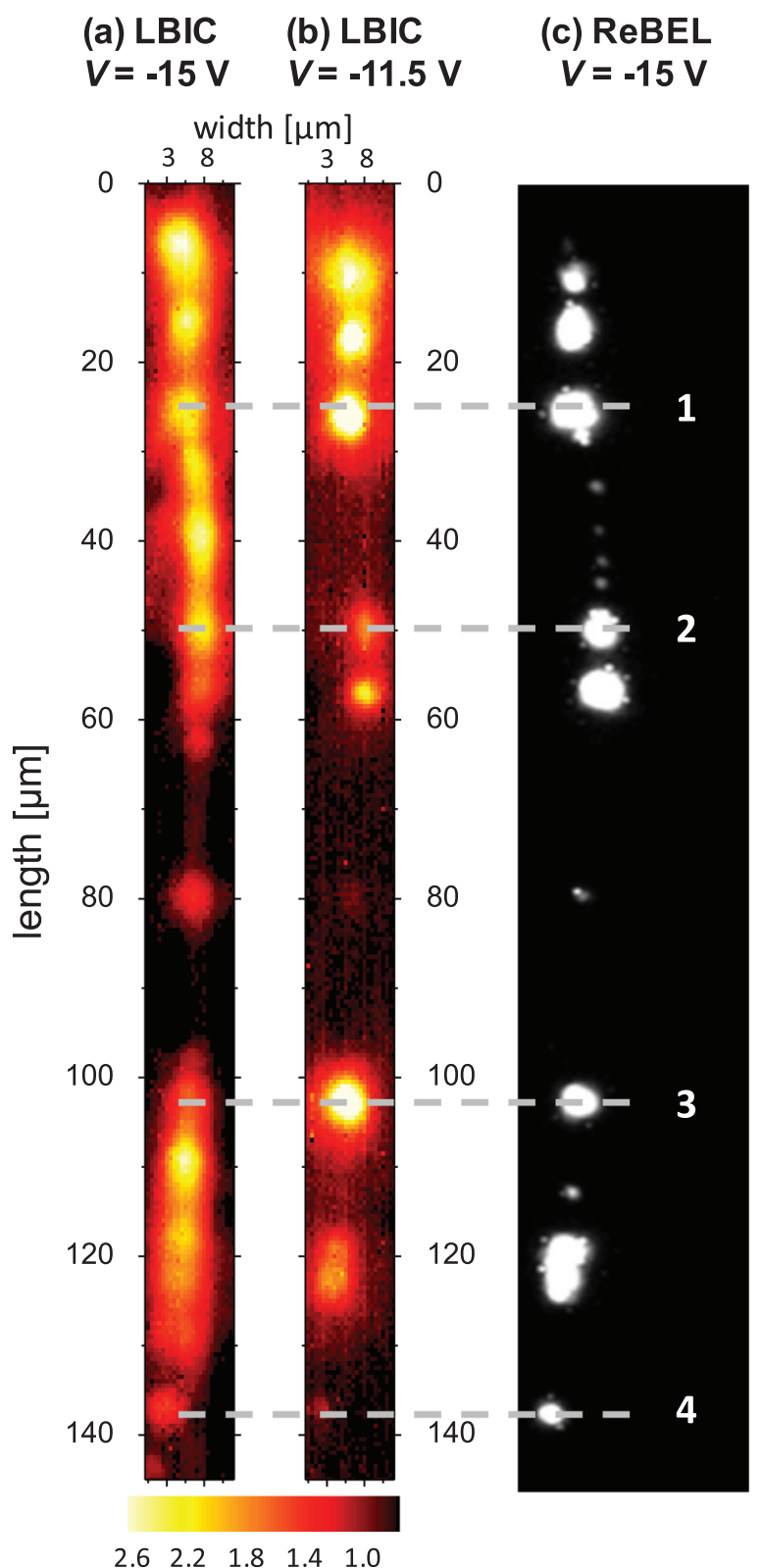

multiplication factor $M$

FIG. 3. (a) An LBIC measurement of a type-2 spot series at an applied voltage $V=-15 \mathrm{~V}$. The respective ReBEL image, pictured with enhanced contrast (c) taken also at $V=-15 \mathrm{~V}$, shows only a rough correlation to the LBIC result in (a). However, if the applied voltage is reduced to the extent to which the spots are barely detected anymore $(V=-11.5 \mathrm{~V})$, the LBIC image (b) shows a perfect correlation to (c). It becomes clear that laser generated carriers are only multiplied in breakdown sites, which are very close to their onset voltage. The four spots (marked 1-4) are investigated further.

until the ReBEL spots of interest are hardly detectable. The resulting LBIC representation (b) now shows an excellent correlation to the ReBEL image. The four marked spots 1-4 were chosen to investigate the dependence of the multiplication factor $M$ on the applied voltage $V$ in more detail.

Fig. 4(a) shows the multiplication factor $M$ of the four breakdown sites chosen in Fig. 3, plotted versus the applied voltage $V$. At lower absolute voltages, the carrier multiplication $M$ is slightly above unity and rises until the gradient of the spot current reaches its maximum. As depicted in Fig.

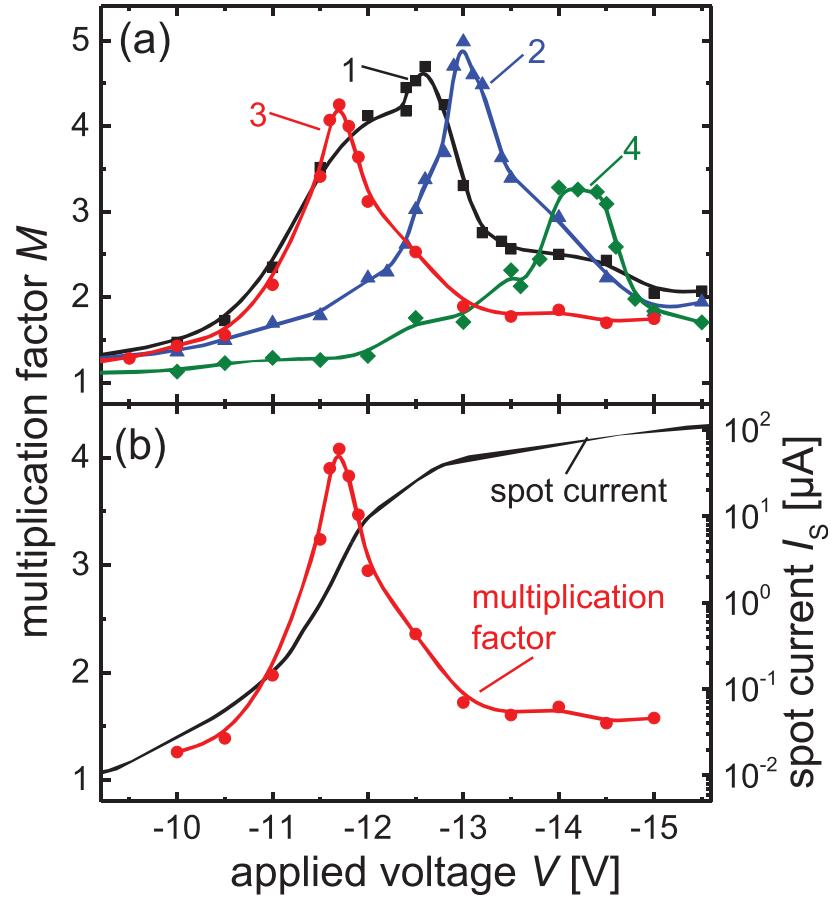

FIG. 4. (a) The four type-2 spots (cf. Fig. 3) show relatively sharp maxima of their measured carrier multiplication $M$ above their onset voltage $V_{\mathrm{O}}$. (b) A comparison of the multiplication factor with the respective spot current $I_{\mathrm{S}}$ (plotted on a logarithmic scale) shows a growth of both values until the spot current becomes high enough and the series resistance limitation sets in. The spot current is linearized, and the measured multiplication factor $M$ drops to a constant value of 1.5 .

4(b), the spot current $I_{\mathrm{S}}$ and multiplication factor $M$ increase rapidly together until the current limitation of the series resistance $R_{\mathrm{S}}$ takes effect. The spot current $I_{\mathrm{S}}$ becomes linear for $V<-12.5 \mathrm{~V}$, the carrier multiplication $M$ has a maximum and then drops to values between $1.5 \leq M \leq 2$. Note that the additional amplified photocurrent also causes additional ReBEL, which is proportional to the carrier multiplication $M$.

The same behaviour is found for type- 3 breakdown sites caused by etch pits on cells with acidic texture. However, no significant multiplication is found for type- 1 breakdown sites caused by aluminium contaminations on the cell surface.

\section{Multiplication factor vs. reference photocurrent}

For the results presented above, the reference photocurrent $I_{\mathrm{Ph} \text {,ref }}$ was about as high as the breakdown current $I_{\mathrm{S}}$ without illumination at the applied voltage $V_{\max }$ of the maximum multiplication $M_{\max }$. Due to the series resistance limitation of the overall current through the breakdown, it is expected that the amplitude of the photocurrent influences the measured carrier multiplication $M$. We therefore sequentially reduced the laser intensity with suitable neutral density filters and repeated the scans of the defect with the applied voltage $V=V_{\max }$. Note that a reduced laser intensity, i.e., reference photocurrent $I_{\mathrm{Ph} \text {,ref }}$ requires a longer measurement

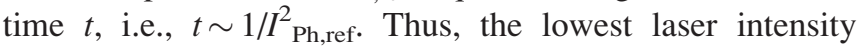
where measurements were possible within a reasonable time $(\approx 2$ days, $5 \times 5$ data points $)$ still corresponds to an illumination of roughly 10 suns. 


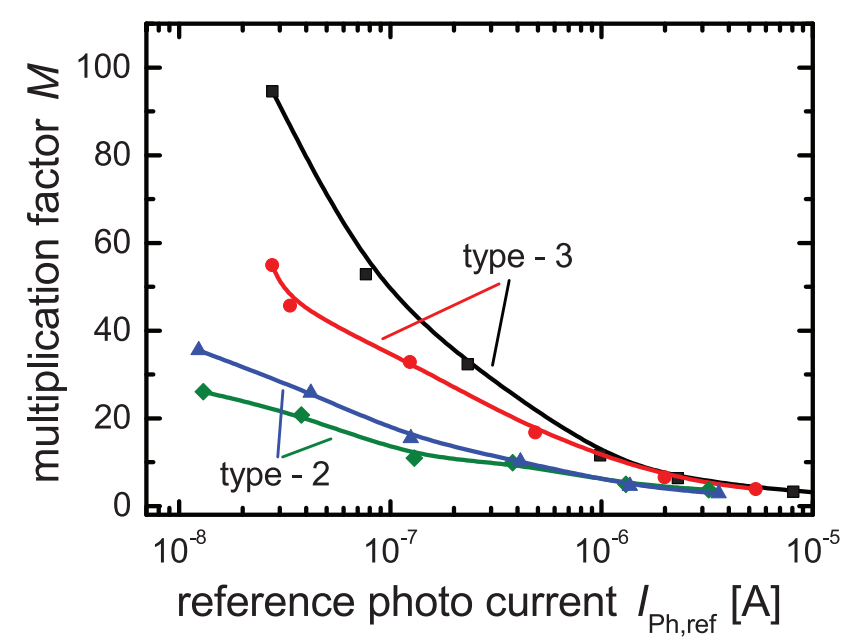

FIG. 5. Multiplication factor $M$-photocurrent $I_{\mathrm{Ph}, \text { ref }}$-diagram of exemplary type- 2 and type- 3 sites. The light-generated- and afterwards amplified current is limited by the local series resistance $R_{\mathrm{S}}$. The illumination intensity is reduced with neutral density filters. All graphs in this diagram show an increase of the multiplication factor $M$ towards smaller reference photo currents $I_{\mathrm{Ph}, \mathrm{ref}}$. The highest measured amplification is $M=94$ for type-3 and $M=35$ for type-2, but no saturation is reached towards lower $I_{\mathrm{Ph}, \text { ref }}$.

Fig. 5 shows the measured multiplication factor $M$ of two type- 2 and type- 3 breakdown sites versus the light generated reference current $I_{\mathrm{Ph}, \text { ref. }}$. As the laser intensity or rather the reference photocurrent $I_{\mathrm{Ph} \text {,ref }}$ is reduced by three orders of magnitude (logarithmic X-axis), the measured multiplication factor $M$ rises continuously to values as high as $M=94$ for type-3 and $M=35$ for type- 2 breakdown sites. Though we found higher multiplication factors for type-3 spots, more measurements would be needed to draw a general conclusion of stronger carrier multiplication for breakdown type-3.

\section{EMPIRICAL MODEL}

In this section, we develop an empirical model to explain the dependences of the measured multiplication factor $M$ on the applied voltage $V$ and on the reference photocurrent $I_{\mathrm{Ph} \text {,ref. }}$ In the dark, we assume the breakdown current $I_{\mathrm{d}}$ to sustain an exponential amplification of the reverse leakage current $I_{0}$ regarding the applied voltage $V$

$$
\left|I_{\mathrm{d}}\right|=I_{0} \exp \left[b\left(-V-R_{\mathrm{S}} I_{\mathrm{d}}+V_{\mathrm{O}}\right)\right]
$$

Note that the onset of the exponential behaviour takes place at the onset voltage $V_{\mathrm{O}}$ and is limited by a series resistance $R_{\mathrm{S}}$. The exponential constant $b$ describes the hardness of the breakdown site. The result provided by the lock-in amplifier is the difference between dark current $I_{\mathrm{d}}$ and illuminated defect current $I_{\mathrm{i}}$ plus the not amplified share of the photo current

$$
\left|I_{\mathrm{i}}\right|+(1-a) I_{\mathrm{Ph}, \mathrm{ref}}-\left|I_{\mathrm{d}}\right|=M I_{\mathrm{Ph}, \text { ref }},
$$

which is by definition the product of injected reference photocurrent $I_{\mathrm{Ph} \text {,ref }}$ and the measured carrier multiplication factor $M$.
Our model adapts the lock-in principle by adding a parallel current source to the dark current, when illuminated [Fig. 2(b)]. We assume that the equivalent circuit of the breakdown site in the dark is a series resistance limited diode, described by Eq. (5). For the illuminated case, we have to add a parallel current path in the model [Fig. 2(b)] with another series resistance $R_{\mathrm{S} 2}$ to fit both measurements ( $M$ vs. $I_{\mathrm{Ph} \text {,ref }}$ and $M$ vs. $V$ ) with the same parameter set. The illuminated current can now be modeled as

$$
\begin{aligned}
I_{\mathrm{i}}= & \underbrace{I_{0} \exp \left[b\left(-V-R_{\mathrm{S}} I_{\mathrm{i}}+V_{\mathrm{O}}\right)\right]}_{\tilde{I}_{\mathrm{d}}} \\
& +a I_{\mathrm{Ph}, \text { ref }} \exp \left\{b_{1}\left[-V-R_{\mathrm{S}} I_{\mathrm{i}}+V_{\mathrm{O}}-R_{\mathrm{S} 2}\left(I_{i}-\tilde{I}_{\mathrm{d}}\right)\right]\right\} .
\end{aligned}
$$

$\tilde{I}_{\mathrm{d}}$ represents the current through the diode under illumination, which differs from the current in the dark by the different voltage drop over the series resistance $\left(I_{\mathrm{i}} R_{\mathrm{S}}\right.$ vs. $\left.I_{\mathrm{d}} R_{\mathrm{S}}\right)$. The first modeling step acquires the parameters of the breakdown site in the dark $b, I_{0}$, and $R_{\mathrm{S}}$ from the respective measured current-voltage characteristic [see Fig. 4(b)] using Newton iteration. Continuing with the same numerical tools, Eq. (7) is evaluated for varying reference photocurrents $I_{\mathrm{Ph}, \text { ref }}$ at the experimentally determined voltage of maximum multiplication $V_{\text {max }}$. Figure 6(a) shows a very good
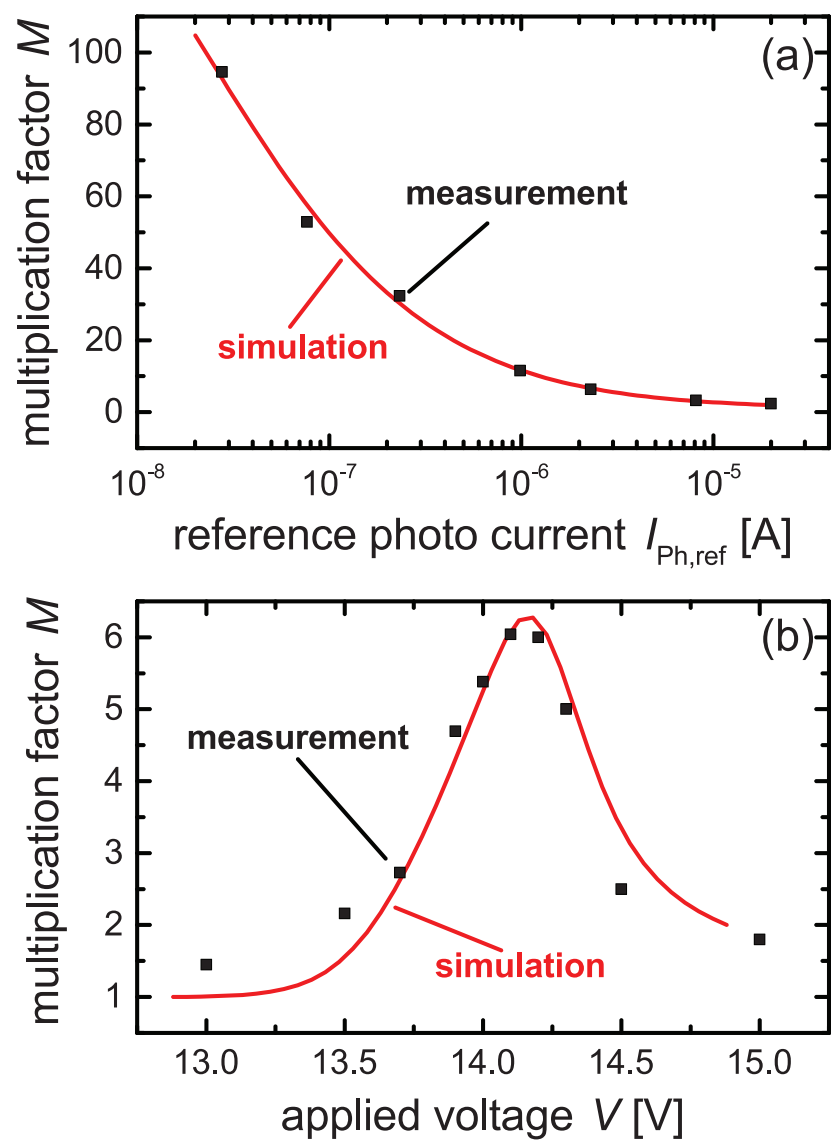

FIG. 6. (a) Comparison of the measured multiplication factor $M$ and its modeled dependence on the reference photocurrent shows an excellent agreement of both curves. (b) Using minor modifications, the same fit parameters achieve a good reproduction of the behavior of $M$ vs. the applied voltage $V$. 
TABLE I. First row: fit parameters for the simulation regarding reference photocurrent $I_{\mathrm{Ph} \text {,ref }}$ depicted in Fig. 6(a): current for the breakdown site at onset voltage $I_{0}$, exponential constants $b$ and $b_{1}$, series resistances $R_{\mathrm{S}}$ and $R_{\mathrm{S} 2}$, and share $a$ of the light impinging on the breakdown site. The second row shows the obtained parameter set for the simulation of the voltage dependence as shown in Fig. 6(b).

\begin{tabular}{lccccc}
\hline \hline$I_{0}[\mathrm{~A}]$ & $b\left[\mathrm{~V}^{-1}\right]$ & $R_{\mathrm{S}}[\mathrm{k} \Omega]$ & $b_{1}\left[\mathrm{~V}^{-1}\right]$ & $R_{\mathrm{S} 2}[\mathrm{k} \Omega]$ & $a$ \\
\hline $8.2 \times 10^{-18}$ & 20.7 & 10.2 & 8.8 & 20 & 0.005 \\
$8.2 \times 10^{-18}$ & 20.7 & 10.2 & 8.4 & 20 & 0.004 \\
\hline \hline
\end{tabular}

agreement between simulation and measurement. The dependence of the multiplication factor $M$ vs. the photocurrent $I_{\mathrm{Ph}}$ is treated first, because it turned out that this part of the simulation is more sensitive to parameter variations than the dependence of $M$ on the applied voltage $V$. The previously acquired parameter set $\left(I_{0}, a, b, b_{1}, R_{\mathrm{S}}\right.$, and $\left.R_{\mathrm{S} 2}\right)$ also reproduces the voltage dependency well [Fig. 6(b)]. However, a shift $\Delta V$ of the modeled peak position $V_{\max }$ on the order of $\Delta V \approx \pm 0.1 \mathrm{~V}$ is necessary. The reason may be that measurements of multiplication factor $M$ and the breakdown current $I_{\mathrm{d}}$ in the dark can hardly be performed at the same time, causing some errors. The parameters for the depicted simulations [Figs. 6(a) and 6(b)] are presented in Table I.

The parameters in the first row of Table I are the ones used for the fit in Fig. 6(a). In order to fit the dependence of the measured multiplication factor $M$ on the applied voltage $V$ [Fig. 6(b)], $b_{1}=8.4 \mathrm{~V}^{-1}$, and $a=0.004$ require minor changes as shown in the second row of Table I.

Using the resulting series resistance $R_{\mathrm{S}}=10.2 \mathrm{k} \Omega$ of the dark current-voltage characteristics to calculate the electrical radius of the respective breakdown site using Eq. (1), yields $r=150 \mathrm{~nm}$. If only the a real overlap between defect and laser $\left(r_{\text {laser }} \approx 1 \mu \mathrm{m}\right.$ ) accounts for the share $a$ of the incoming light which is multiplied, it will be around $a \approx 0.02$. We therefore assume that only roughly a quarter $(a=0.005)$ of carriers generated above and below the defect are travelling through the multiplying region.

\section{DISCUSSION OF THE THERMOGRAPHY METHOD}

The multiplication factor illuminated thermography (MFILIT $)^{2}$ uses pulsed illumination of a reverse-biased solar cell to measure the spatially resolved current in the sample. The multiplication factor is proportional to the ratio of an image at a high reverse bias, for example, $V_{\text {high }}=-15 \mathrm{~V}$ and an image taken at lower reverse bias, for example, $V_{\text {low }}=-13 \mathrm{~V}$, where no ReBEL is seen. However, when a $640 \times 512$-pixel thermo camera images a $(156 \mathrm{~mm})^{2}$ solar cell, the resolution is $10^{4} \mu \mathrm{m}^{2} /$ pixel. A single defect with less than $0.01 \mu \mathrm{m}^{2}$ active area would require a multiplication factor $>10^{6}$ to be detected. Our LBIC method with its spatial resolution of $\approx 4 \mu \mathrm{m}^{2}$ has a significant advantage in terms of detecting defects and therefore should yield even higher multiplication factors than we reported. But defects rarely appear spatially isolated, especially etch pits are observed in large numbers per area. Furthermore, our measurements indicate that etch pits may show LBIC signals without being identified as breakdown sites due to ReBEL emission. A sufficient density of type-3 sites, i.e., etch pits, may be one condition to measure carrier multiplication with MF-ILIT. Note that our preliminary MF-ILIT measurements on the samples later studied by LBIC did never show any carrier multiplication, possibly because of the low number of defect sites per area.

Furthermore, the lowest laser intensity for our LBIC measurements approximately corresponds to 10 suns while MF-ILIT is usually performed at 0.1 sun. Indeed, Figs. 5 and 6 show that the carrier multiplication factor $M$ increases strongly when reducing the light intensity. However, our model indicates a saturation of the measured multiplication factor around $M \approx 150$ at approximately 1 sun illumination. According to the estimation given above, still a density of defect sites on the order of $10^{4} /$ cell area projected onto a pixel is required for measuring multiplication factors around 4 as reported for the MF-ILIT method.

\section{CONCLUSIONS}

The high spatial resolution of an LBIC scanning method using a focused laser beam allows the investigation of carrier multiplication of single ReBEL spots. We directly measure multiplication $M$ of the injected photocurrent with factors up to 35 (type-2) and 94 (type-3), and no multiplication for type-1 breakdown sites. We successfully model our measurements using two parallel, series resistance limited diodes, which yield the share $a$ of the injected and multiplied photocurrent. This share must be on the order of $a=1 / 200$, according to the simulations. It is, therefore, reasonable to suggest that the actual multiplication factors $m=M / a$ are at least around 5000, especially for type-2 breakdown sites, which were so far assumed not to exhibit carrier multiplication but only Zener tunneling. We do not deny the existence of Zener tunneling for type-2 sites as an initial process, but our results show that it is followed by avalanche multiplication. The discrepancy of our results to previous methods like lock-in thermography is mainly explained with an insufficient spatial resolution of previous method.

\section{ACKNOWLEDGMENTS}

The authors thank Thomas C. M. Müller, Josef Klomfass, Markus Hülsbeck, Yael Augarten, and Bart E. Pieters (Jülich) for help with the experiments and fruitful discussions. The authors are also grateful to O. Breitenstein (MPI Halle) for various helpful discussion.

${ }^{1}$ Y. Kaji, H. Kondo, Y. Takahashi, T. Yamazaki, Y. Uraoka, and T. Fuyuki, in Proceedings of the 31st IEEE Photovoltaic Specialists Conference (IEEE, Piscataway, NJ, 2005), pp. 1346-1348.

${ }^{2}$ O. Breitenstein, J. Bauer, J.-M. Wagner, and A. Lotnyk, Prog. Photovolt. 16, 679 (2008).

${ }^{3}$ D. Lausch, K. Petter, H. V. Wenckstern, and M. Grundmann, Phys. Status Solidi RRL 3, 70 (2009).

${ }^{4}$ D. Lausch, K. Petter, R. Bakowski, C. Czekalla, J. Lenzner, H. V. Wenckstern, and M. Grundmann, Appl. Phys. Lett. 97, 073506 (2010).

${ }^{5}$ W. Kwapil, P. Gundel, M. C. Schubert, F. D. Heinz, W. Warta, E. R. Weber, A. Goetzberger, and G. Martinez-Criado, Appl. Phys. Lett. 95, 232113 (2009).

${ }^{6}$ A. Hähnel, J. Bauer, H. Blumtritt, O. Breitenstein, D. Lausch, and W. Kwapil, J. Appl. Phys. 113, 044505 (2013).

${ }^{7}$ J. Bauer, J. M. Wagner, A. Lotnyk, H. Blumtritt, B. Lim, J. Schmidt, and O. Breitenstein, Phys. Status Solidi RRL 3, 40 (2009). 
${ }^{8}$ O. Breitenstein, J. Bauer, K. Bothe, W. Kwapil, D. Lausch, U. Rau, J. Schmidt, M. Schneemann, M. C. Schubert, J.-M. Wagner, and W. Warta, J. Appl. Phys 109, 071101 (2011).

${ }^{9}$ R. Newman, Phys. Rev. 100, 700 (1955).

${ }^{10}$ A. G. Chynoweth and K. G. McKay, Phys. Rev. 102, 396 (1956).

${ }^{11}$ A. Goetzberger and W. Shockley, J. Appl. Phys. 31, 1821 (1960).

${ }^{12}$ J. Bauer, D. Lausch, H. Blumtritt, N. Zakharov, and O. Breitenstein, Prog. Photovolt. 21, 1444 (2013).
${ }^{13}$ S. M. Sze and G. Gibbons, Solid State Electron. 9, 831 (1966).

${ }^{14}$ M. Schneemann, T. Kirchartz, R. Carius, and U. Rau, J. Appl. Phys. 114, 134509 (2013).

${ }^{15}$ M. Schneemann, A. Helbig, T. Kirchartz, R. Carius, and U. Rau, Phys. Status Solidi A 207, 2597 (2010).

${ }^{16}$ R. G. Mazur and D. H. Dickeya, J. Electrochem. Soc.113, 255 (1966).

${ }^{17}$ R. Holm, Electric Contacts Handbook (Springer, Berlin, 1958).

${ }^{18}$ J. C. Irvin, Bell Syst. Tech. J. 41, 387 (1962). 